\title{
Sensitivity Enhancement of a Surface Plasmon Resonance Sensor with Platinum Diselenide
}

\author{
Yue Jia ${ }^{1,+}$, Zhongfu Li ${ }^{1,+}$, Haiqi Wang ${ }^{1}$, Muhammad Saeed ${ }^{2}$ and Houzhi Cai ${ }^{1, *}$ \\ 1 College of Physics and Optoelectronic Engineering, Shenzhen University, Shenzhen 518060, China; \\ onlyjiayue@hotmail.com (Y.J.); lzf_857948523@outlook.com (Z.L.); whq143@outlook.com (H.W.) \\ 2 Institute for Advanced Study, Shenzhen University, Shenzhen 518060, China; saeedphysics96@gmail.com \\ * Correspondence: hzcai@szu.edu.cn \\ + These two authors have equal contributions.
}

Received: 10 November 2019; Accepted: 17 December 2019; Published: 24 December 2019

check for updates

\begin{abstract}
The extraordinary optoelectronic properties of platinum diselenide $\left(\mathrm{PtSe}_{2}\right)$, whose structure is similar to graphene and phosphorene, has attracted great attention in new rapidly developed two-dimensional (2D) materials beyond the other 2D material family members. We have investigated the surface plasmon resonance (SPR) sensors through $\mathrm{PtSe}_{2}$ with the transfer matrix method. The simulation results show that the anticipated $\mathrm{PtSe}_{2}$ biochemical sensors have the ability to detect analytic. It is evident that only the sensitivities of Ag or Au film biochemical sensors were observed at $118^{\circ} / \mathrm{RIU}$ (refractive index unit) and $130^{\circ} / \mathrm{RIU}$, whereas the sensitivities of the $\mathrm{PtSe}_{2}$-based biochemical sensors reached as high as $162^{\circ} / \mathrm{RIU}$ (Ag film) and $165^{\circ} / \mathrm{RIU}$ (Au film). The diverse biosensor sensitivities with $\mathrm{PtSe}_{2}$ suggest that this kind of 2D material can adapt SPR sensor properties.
\end{abstract}

Keywords: biosensor; Kretschmann; $\mathrm{PtSe}_{2}$; SPR; transition metal dichalcogenides (TMDCs)

\section{Introduction}

We have investigated biochemical sensors based on the surface plasmon resonance technique, which has been applied in extraordinary biological and chemical applications, because of their biocompatibility, excellent sensitivity, and accurate detection for pharmaceutics, for instance, in medical diagnostics and enzyme detection [1-4]. Surface plasmon resonance (SPR) refers to the resonance excitation of the surface plasmon polaritons (SPPs) at the interface of the negative (metal) and positive (dielectrics) constant materials, which is considered as best suited for untagging sensing and real-time monitoring [5,6].

One of the most effective ways for improving the efficiency of sensing devices is to select a material, such as graphene, to optimize sensing functions [7-10]. The significant properties of transition metal dichalcogenide (TMDC) materials, such as their absorption rate $(\sim 5 \%)$, which is higher compared to a graphene monolayer $(2.3 \%)$, entirely different large tunable band gap than the zero band gap of graphene, and large biosense work function in comparison to graphene, are increasingly becoming preferred in biosensing applications. The outstanding electrical, optical, and chemical properties of TMDCs are converting them as promising candidates and accessory materials in comparison to graphene for the future generation of electronic and optic facilities [11-13]. TMDC-based SPR sensors are recommended for refractive index sensing and exhibit highly improved sensitivity $[14,15]$.

A series of ultrasensitive photonic crystal fiber based surface plasmon resonance fiber sensors have been made by Md. Saiful Islam et al. proposed polarization-sensitive sensors that showed a relatively high wavelength sensitivity of 25,000 nm/RIU and a high detection limit [16-18]. Wu et al. obtained the highest sensitivity of $134.6^{\circ} /$ RIU by using a graphene layer for the SPR biosensor to enhance the sensitivity caused by the light absorbed $[15,19]$. L. Wu and $\mathrm{Z}$. Lin et al. have produced $\mathrm{MoS}_{2}-$ graphene 
hybrid structures that are biocompatible and useful in the field of biosensors. Maximum sensitivities of $182^{\circ} / \mathrm{RIU}$ and $190^{\circ} / \mathrm{RIU}$ were obtained with 4-layer $\mathrm{MoS}_{2}$ and a monolayer of graphene or 6-layer $\mathrm{MoS}_{2}$ coatings, respectively, both on surfaces of Al thin film [20,21]. Ouyang et al. reported that PR biosensors using $\mathrm{MoS}_{2}$ to improve the sensitivity had the highest sensitivity of $125^{\circ} / \mathrm{RIU}$ [22].

As single-layer $\mathrm{PtSe}_{2}$ exhibits the same pattern of structure as graphene and phosphorene, it also keeps excellent optical and electrical properties that have attracted great attention as a $2 \mathrm{D}$ material beyond the predecessor members [10,23,24]. $\mathrm{PtSe}_{2}$ is a group ten TMDC with a 1T-phase [25]. The band gap of $\mathrm{PtSe}_{2}$ is highly tunable because of its intrinsic quantum confinement effect and strong interlayer interaction. This leads to a type-II Dirac semimetal-to-semiconductor transition when going from bulk to few-layer form and exhibits the largest band gap of $\sim 1.2 \mathrm{eV}$ for monolayer (ML) $\mathrm{PtSe}_{2}$ (from theoretical prediction) [26,27]. Moreover, different types of stress can be applied on $\mathrm{PtSe}_{2}$, which can be modulated easily [28]. Furthermore, less toxicity and chemical stability has been investigated in $\mathrm{PtSe}_{2}$ sensing applications [23,29,30]. TMDC $\mathrm{PtSe}_{2}$ monolayers not only possess a significant thermoelectric character, having semiconductor properties, but it also has an outstanding optoelectronic property. To the best of our knowledge, few systematic studies have been performed on the optic and electric properties of $\mathrm{PtSe}_{2}$.

In the visible light region, $\mathrm{Ag}$ and $\mathrm{Au}$ are considered ideal candidates as metallic films [4]. Ag films usually give a sharper peak compared Au and offer better sensitivity, which is used for enhancing biosensor sensitivity [31]. In the reaction system, however, the Au film shows stability, strong adhesion with the glass, and it produces no reaction with inorganic ions. As Au is not susceptible to oxidation, and it usually does not react with most chemicals, it is usually used as metallic film in sensors [32]. Here, $\mathrm{Ag}$ and $\mathrm{Au}$ film is used as a metallic layer coating on top of the BK7 substrate in SPR biological systems. The $2 \mathrm{D}$ material $\mathrm{PtSe}_{2}$ might be used as the protective layer adjoining the biomolecular recognition elements to prevent oxidation and increase biomolecule adsorption. In this paper, the 2D material $\mathrm{PtSe}_{2}$ is used in SPR biochemical sensors to enhance the sensitivity and retain chemical stability. Here, Kretschmann's attenuated total reflection (ATR) configuration has been selected as the SPR sensor structure [33]. Kretschmann's configuration is one of the most basic SPR configurations of biosensors and is usually composed of a coupling prism and metallic film. Biomolecules and metallic membranes can interact with each other, through which biomolecules can be detected. We have used Ag or Au to cover, on the basis of the optical coupling prism, BK7 glass to be used as the coupling prism, and PtSe 2 covers the metallic layer in this structure [34,35].

\section{Calculation Models and Methods}

Z. Lou and other authors provided their synthesized nanoparticle-organic clusters (NOCs) as signal amplification reagents permitting an SPR signal four times higher than that of the sandwich format [36-38]. Here, our design presents a proposed structure having a high-sensitivity sensor separately containing $\mathrm{PtSe}_{2}$, as shown in Figure 1. In these SPR structures, the Ag or Au film is installed on top of the BK7 coupling prism, and the $\mathrm{PtSe}_{2}$ layer is the biomolecular recognition element coated on the $\mathrm{Ag} / \mathrm{Au}$ film surface. The size of the thickness of the $\mathrm{PtSe}_{2}$ monolayer is $0.375 \mathrm{~nm}$ [27]. We have used BK7 glass as a coupling prism. As the first-rank metal for SPP, the Au film or Ag film is chosen at $50 \mathrm{~nm}$. The BK7 glass refractive index can be calculated using the following equation [39]:

$$
n_{B K 7}=\left(\frac{1.03961212 \lambda^{2}}{\lambda^{2}-0.00600069867}+\frac{0.231792344 \lambda^{2}}{\lambda^{2}-0.0200179144}+\frac{1.03961212 \lambda^{2}}{\lambda^{2}-103.560653}+1\right)^{\frac{1}{2}}
$$

The refractive index of $\mathrm{Ag}$ or $\mathrm{Au}$ can be expressed from the Drude-Lorentz model [40]:

$$
\mathrm{n}_{m}=\sqrt{\varepsilon_{m}}=\left[1-\frac{\lambda^{2} \lambda_{c}}{\lambda_{p}^{2}\left(\lambda_{c}+i \lambda\right)}\right]^{\frac{1}{2}}
$$


where $\lambda_{c}$ and $\lambda_{p}$ represent the collision and plasma wavelengths. The values of $\lambda_{c}$ and $\lambda_{p}$ for Ag are $1.7614 \times 10^{-5} \mathrm{~m}$ and $1.4541 \times 10^{-7} \mathrm{~m}$, whereas for Au they are $8.9342 \times 10^{-6} \mathrm{~m}$ and $1.6826 \times 10^{-7} \mathrm{~m}$, respectively. In order to further enhance the sensibility of the designed biochemical sensors, we have covered the metal Ag or Au film surface with $\mathrm{PtSe}_{2}$ to impede the metal being oxidized.

Experimental modulating is used to get the refractive indices as a variable quantity of $\mathrm{PtSe}_{2}$, which is composed of real and imaginary parts [41]. The real and imaginary parts of the complex dielectric function of $\mathrm{PtSe}_{2}$ for a wavelength at $633 \mathrm{~nm}$ are exhibited in Figure 2. The expression $n_{s}=1.33+\Delta n$ is presented as the refractive index in the sensitive medium, and $\Delta n$ represents refractive index change in sensitive medium as a result of biological action or a chemical reaction.

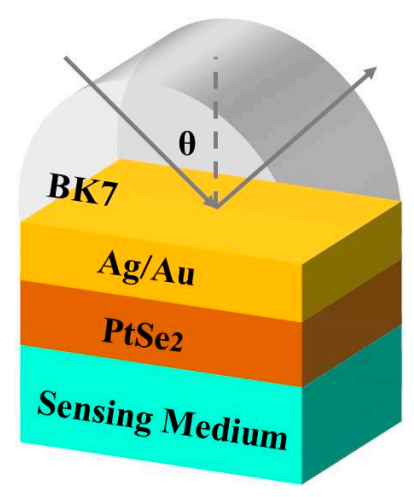

Figure 1. Sketch diagram of the $\mathrm{PtSe}_{2}$ surface plasmon resonance (SPR) biochemical sensor to enhance the sensitivity.

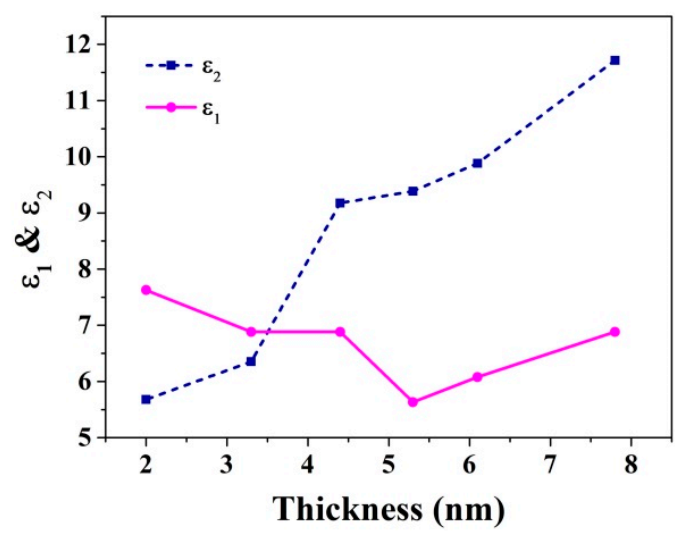

Figure 2. Real and imaginary parts of the complex dielectric function for $\mathrm{PtSe}_{2}$ at a wavelength of $633 \mathrm{~nm}$.

The transfer matrix method has been used [42] to investigate the reflectivity of the incident TM-polarized light of the N-layer model, as the matrix method is accurate and without approximations. MATLAB was used to compute the analogous SPR modulation. In these $\mathrm{PtSe}_{2}$ biosensors, all layers are piled in the direction of the vertical BK7 glass coupling prism, and each layer is named by the refractive index $\left(n_{k}\right)$, thickness $\left(d_{k}\right)$, and dielectric constant $\left(\varepsilon_{k}\right)$, respectively. The resonance angle is the minimum reflectance corresponding to the incident angle. The tangential field of the first boundary $Z=Z_{1}=0$ is correlated with the tangential field of the final boundary $Z=Z_{N-1}$ :

$$
\left[\begin{array}{l}
U_{1} \\
V_{1}
\end{array}\right]=M\left[\begin{array}{l}
U_{N-1} \\
V_{N-1}
\end{array}\right]
$$


where $V$ and $U$ represent the components of magnetic and electric fields at the limiting surface. $M$ and $k$ present the characteristic matrix of the composite architecture and the $k$ th layer in the $N$-layered model, respectively. P-polarized light is made up of:

$$
\begin{gathered}
\prod_{K=2}^{N-1} M_{K}=\left[\begin{array}{ll}
M_{11} & M_{12} \\
M_{21} & M_{22}
\end{array}\right], \\
M_{K}=\left[\begin{array}{cc}
\cos \beta_{K} & \frac{-\sin \beta_{K}}{q_{k}} \\
-\mathrm{i} \mathrm{q}_{k} \sin \beta_{k} & \cos \beta_{k}
\end{array}\right], \\
\mathrm{q}_{k}=\left(\frac{\mu_{k}}{\delta_{k}}\right)^{\frac{1}{2}} \cos \theta_{k}=\frac{\left(\varepsilon_{k}-n_{1}^{2} \sin \theta_{1}^{2}\right)^{\frac{1}{2}}}{\varepsilon_{k}}, \\
\beta_{\mathrm{k}}=\frac{2 \pi}{\lambda} n_{k} \cos \theta_{k}\left(Z_{k}-Z_{k-1}\right)=\frac{2 \pi d_{k}}{\lambda}\left(\varepsilon_{k}-n_{1}^{2} \sin \theta_{1}^{2}\right)^{\frac{1}{2}} .
\end{gathered}
$$

After mathematical simplifications, we get the p-polarized light $N$-layer complex reflection coefficient $r_{p}$, and the corresponding amplitude reflection coefficient $\left(R_{p}\right)$ might be obtained by the square of $r_{p}$ :

$$
\begin{gathered}
r_{p}=\frac{\left(M_{11}+M_{12} q_{5}\right) q_{1}-\left(M_{21}+M_{22} q_{5}\right)}{\left(M_{11}+M_{12} q_{5}\right) q_{1}+\left(M_{21}+M_{22} q_{5}\right)}, \\
R_{p}=\left|r_{p}\right|^{2} .
\end{gathered}
$$

The transformation of sensing medium in refractive index $(\Delta n)$ can initiate the alteration of the resonance angle $(\Delta \theta)$, and the sensitivity can be indicated as SR1 $=\Delta \theta / \Delta n$ [43].

The detection accuracy (DA) is the ratio in the reflectance curve of shift in resonance angle $\left(\Delta \theta_{\text {res }}\right)$ to the full width at half-maximum (FWHM):

$$
D A=\Delta \theta / F W H M
$$

The figure of merit (FOM) is the ratio in the reflectance curve of sensitivity (S) to the FWHM [44]:

$$
F O M=S / F W H M
$$

\section{Results and Discussion}

Because of the low refractive index of BK7 glass, it was chosen as the coupling prism in these expected biochemical sensors. For the conventional SPR, the Kretschmann geometry biochemical sensor mostly offers a simplex metallic layer to survey SPP, as illustrated in Figure 3. The values of the sensitivity of the conventional Ag or Au metal were $118^{\circ} / \mathrm{RIU}$ and $130^{\circ} / \mathrm{RIU}$, as shown in Figure $3 \mathrm{a}, \mathrm{C}$, respectively, by using BK7 glass prism. However, this value is not satisfactory for biochemical sensor sensitivity $[45,46]$. In this paper, we have developed SPR biochemical sensors by using 2D TMDC $\mathrm{PtSe}_{2}$ materials to enhance the sensitivities of the sensors.

The addition of the 2D TMDC $\mathrm{PtSe}_{2}$ material between the metallic (Au or Ag) film and sensing medium in the structure of biosensors increased the sensitivities. The results clearly suggest a large improvement in the sensitivities when the $\mathrm{PtSe}_{2}$ monolayer covers the previous structure, as shown in Figure 3b,d. For the Ag film or Au film, we have obtained sensitivities equal to $162^{\circ} / \mathrm{RIU}$ and $165^{\circ} / \mathrm{RIU}$ at the layers accumulating 16 and 12 layers, respectively.

In future experiments, $2 \mathrm{D} \mathrm{PtSe}{ }_{2}$ nanosheets can be prepared by the liquid-phase exfoliation (LPE) method, and then the nanosheets will be coated on the Ag or Au thin film ( 50 nm) with the BK7 substrate by using the spin coating method [47]. The SPR sensors can be constructed by connecting the $\mathrm{PtSe}_{2}-\mathrm{Ag} / \mathrm{Au}-\mathrm{BK} 7$ multilayer films and the BK7 prism with the refractive index matching solution. 
Based on these biochemical sensor results, the 2D TMDC $\mathrm{PtSe}_{2}$ material might increase sensitivities. The reflectivity of $\mathrm{PtSe}_{2}$ layers changed with the variation in the incident angle, which is shown in Figure $4 \mathrm{a}, \mathrm{c}$, illustrating that sensitivities varied with different layers of $\mathrm{PtSe}_{2}$-based biochemical sensors from $n_{s}=1.33$ to $n_{s}=1.37$ to the sensing medium refractive index. The reflectivity changes with the incident angle are shown in Figure $4 b$,d. It is clear from Figure $4 b$,d that, with the increase of refractive indices of the sensitive media, the angles of resonance of $\mathrm{PtSe}_{2}$ sensors at $2.0 \mathrm{~nm}$ thickness shifted to a higher angle of incidence. The resonance angles were $67.54^{\circ}, 68.13^{\circ}, 68.75^{\circ}, 69.39^{\circ}$, and $70.06^{\circ}$ when $n_{s}=1.33, n_{s}=1.34, n_{s}=1.35, n_{s}=1.36$, and $n_{s}=1.37$ for Ag film, respectively. Whereas, the resonance angles for Au film were $70.33^{\circ}, 71.01^{\circ}, 71.73^{\circ}, 72.49^{\circ}$, and $73.28^{\circ}$ when $n_{s}=1.33, n_{s}=1.34, n_{s}=1.35$, $n_{s}=1.36$, and $n_{s}=1.37$, respectively.

Figure 5 a presents distributions of the electric field for the proposed SPR sensors for PtSe ${ }_{2}$ material on Ag or Au film, respectively. It has been investigated that coating $\mathrm{PtSe}_{2}$ on the Ag film might enhance the electric field at the sensor/sensing medium interface, where $\mathrm{PtSe}_{2}$ gets the maximum electric field strength, since $\mathrm{PtSe}_{2}$ appears in the middle and is located at the last single layer of $\mathrm{PtSe}_{2}$.
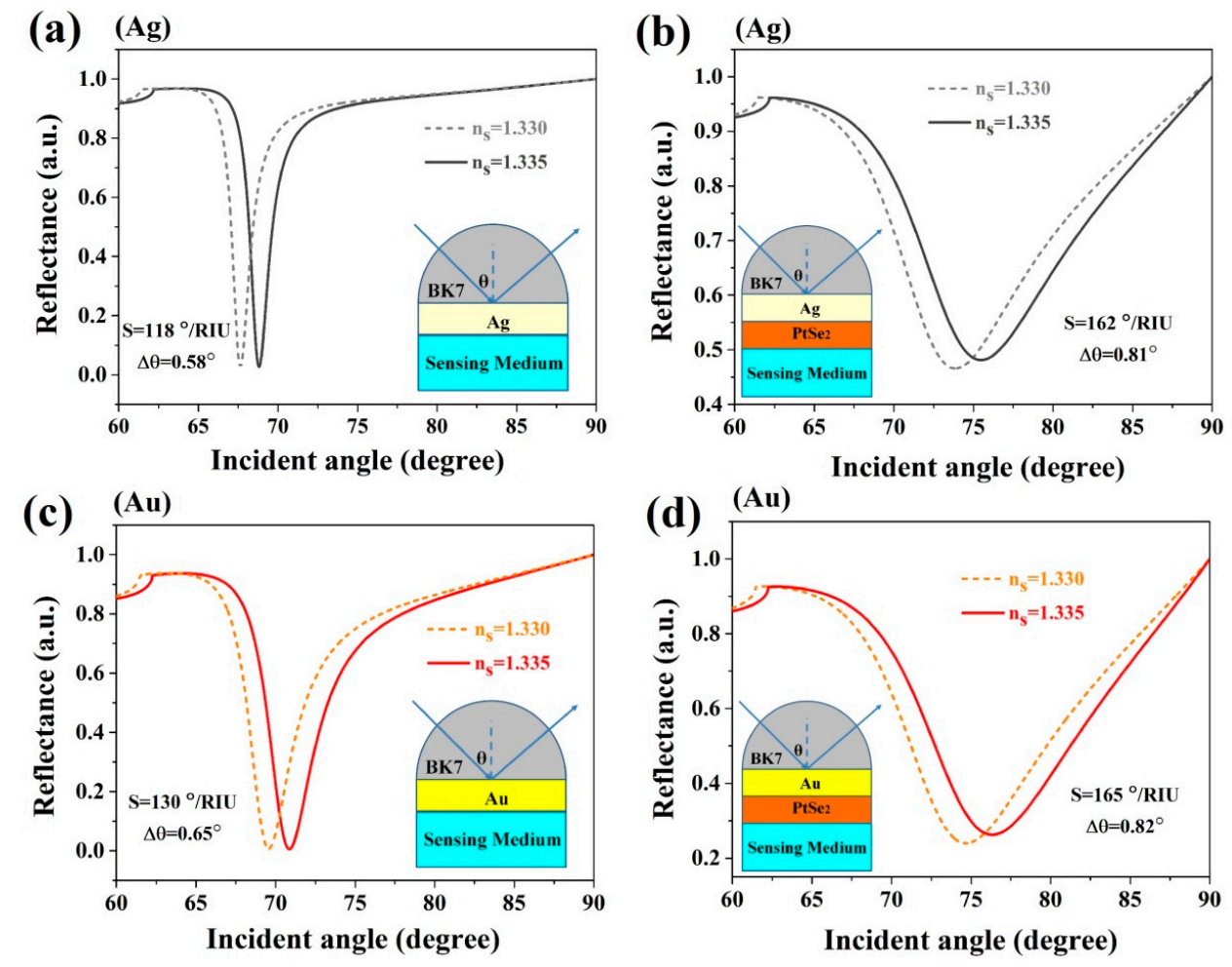

Figure 3. Variation of reflectivity with incident angles for (a),(c) the conventional biochemical sensor based on simplex Ag or Au film; and (b),(d) the proposed biochemical sensors with $\mathrm{PtSe}_{2}$ on $\mathrm{Ag}$ or $\mathrm{Au}$, respectively. 

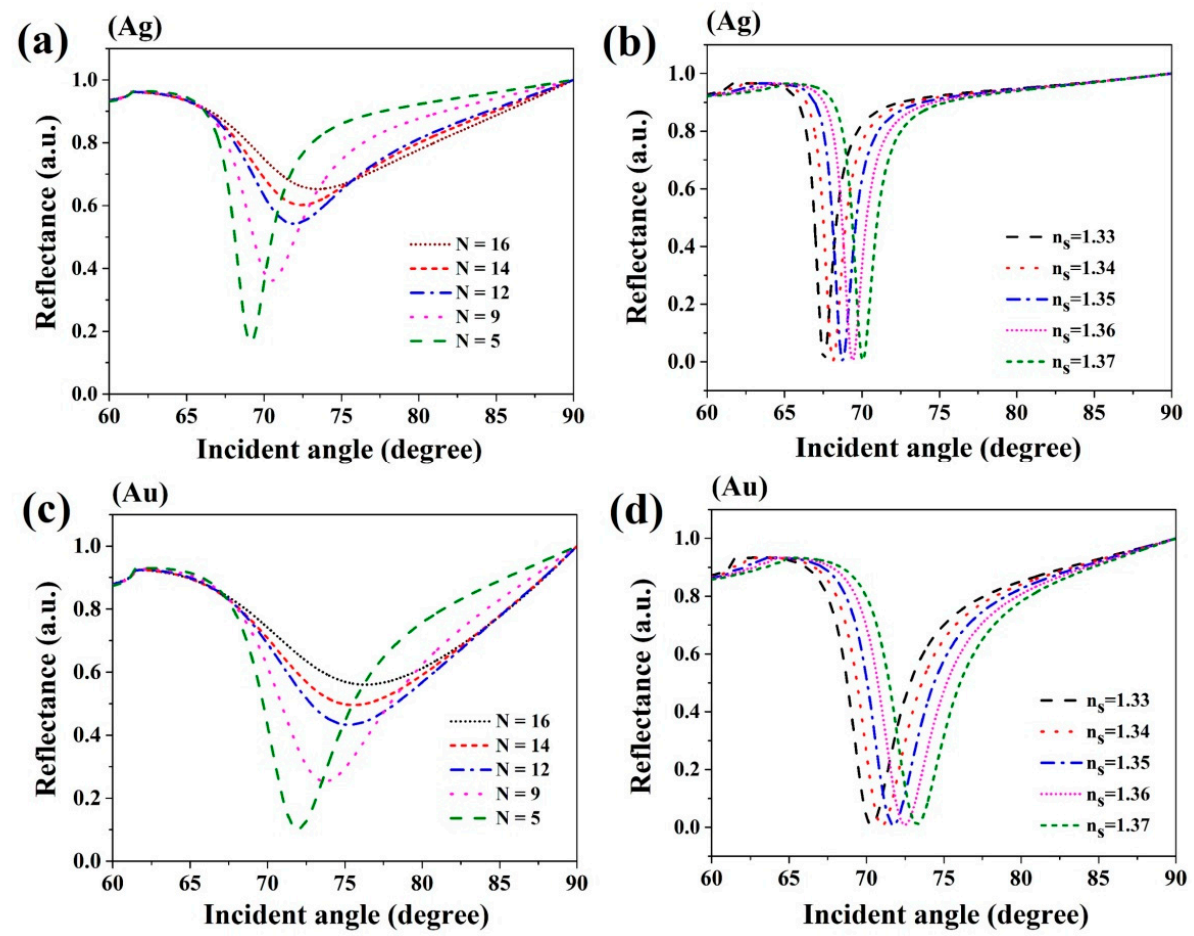

Figure 4. (a,c) The reflectances of the $\mathrm{PtSe}_{2}$ biosensor on $\mathrm{Ag}$ film and $\mathrm{Au}$ film change with different numbers of $\mathrm{PtSe}_{2}$ layers, respectively; $(\mathbf{b}, \mathbf{d})$ the reflectance of $\mathrm{PtSe}_{2}$ biosensor with $2.0 \mathrm{~nm} \mathrm{PtSe}$ varies with the refractive indices of the sensing medium on $\mathrm{Ag}$ film and $\mathrm{Au}$ film, respectively.

(a)

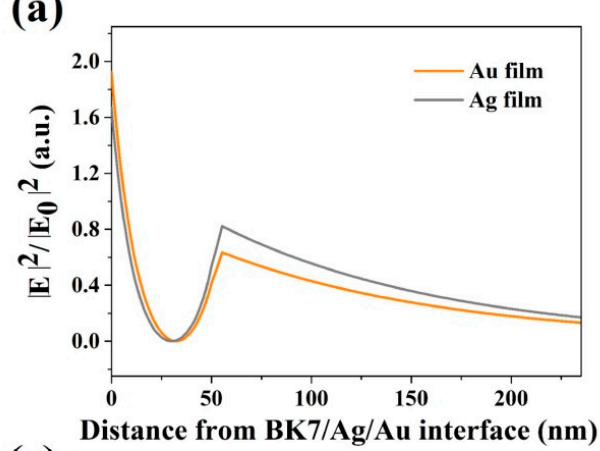

(c)

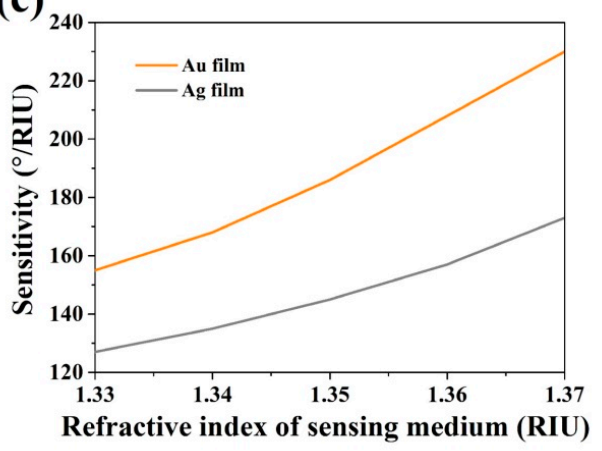

(b)

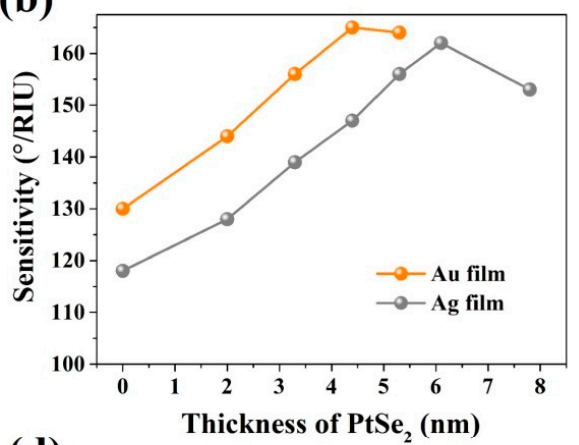

(d)

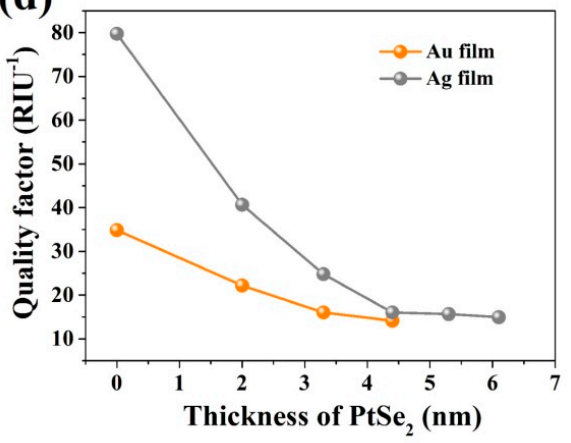

Figure 5. (a) Electric field distributions for the $\mathrm{PtSe}_{2}$ on $\mathrm{Ag}$ film and $\mathrm{Au}$ film sensors; (b) Variation of sensitivity with respect to $\mathrm{PtSe}_{2}$ in different thicknesses; (c) The sensitivities of the proposed biochemical sensors with $2.0 \mathrm{~nm} \mathrm{PtSe}{ }_{2}$ vary with the refractive indices of sensing medium on $\mathrm{Ag}$ film and Au film, respectively; (d) Variation of quality factors with respect to the thickness of $\mathrm{PtSe}_{2}$ varying from 0 to 7 nm of $\mathrm{PtSe}_{2}$ biosensor. 
The changes of sensitivities for $\mathrm{PtSe}_{2}$ on $\mathrm{Ag}$ and Au surface biosensors are plotted in Figure $5 \mathrm{~b}$. It can be seen that the values of the highest sensitivities of $\mathrm{PtSe}_{2}$ occur at $162^{\circ} / \mathrm{RIU}$ and $165^{\circ} / \mathrm{RIU}$, respectively. Then, the sensitivity curves decrease with an increasing $\mathrm{PtSe}_{2}$ layer thickness, causing the gold or silver film coating on the $\mathrm{PtSe}_{2}$ nanosheet overlayer to affect the evanescent electric field distribution, which, in turn, has an impact on the decay length [47]. Figure 5c shows the electric field distributions of $2.0 \mathrm{~nm} \mathrm{PtSe} 2$ on $\mathrm{Ag}$ film and $\mathrm{Au}$ film biochemical sensors, separately. The refractive indices of the inductive media changed within the range of 1.33 to 1.37 , and the change in the electric field of graphene/sensing medium interface has been clearly observed. It was found that the designed $\mathrm{PtSe}_{2}$-based biochemical sensors are sensitive when detecting small changes in sensitive media. A major change of the surface wave characteristics might occur as a result of small changes in the refractive index of sensitive medium close to the interface, which may produce change in the electric field. Figure $5 \mathrm{~d}$ gives the change of FOM for this $\mathrm{PtSe}_{2}$ biosensor. When the thickness of the $2 \mathrm{D} \mathrm{PtSe}_{2}$ material was from 0 to $7 \mathrm{~nm}$, and the change of width was kept from $1^{\circ}$ to $3^{\circ}$, we calculated the FOM using Equation (11). From this calculation, we can determine that the FOM decreases with the increase of thickness on both Ag and Au films.

Table 1 presents the information about change in thickness of $\mathrm{PtSe}_{2}$, layer numbers $(L)$, highest sensitivity $(S)$, minimum resonance angle $\left(\theta_{\text {min }}\right)$ at $n_{s}=1.33$, and resonance angle change $(\Delta \theta)$ from $n_{s}=1.330$ to $n_{s}=1.335$. The smallest reflectivity $\left(R_{\text {min }}\right)$, FWHM, and FOM were at $n_{s}=1.33$, and DA was at $n_{S}=1.33$ to $n_{S}=1.34$ in these PtSe 2 biochemical sensors on $\mathrm{Ag}$ or Au films. The thicknesses of Ag and $\mathrm{Au}$ were set to $50 \mathrm{~nm}$. We set the range of the incident angle from $0^{\circ}$ to $90^{\circ}$. The thicknesses of $\mathrm{PtSe} \mathrm{C}_{2}$ layers came from reference [41], and layer numbers were calculated from the thickness of the $\mathrm{PtSe}_{2}$ monolayer, which was $0.375 \mathrm{~nm}$. The reflectivity curve steps forward at a higher angle with an increase in the number of $\mathrm{PtSe}_{2}$ layers. Once the $\mathrm{PtSe}_{2}$ layers are augmented to the optimized value, maximum sensitivity can be obtained. For $\mathrm{PtSe}_{2}$ on $\mathrm{Ag}$ and $\mathrm{Au}$, the sensitivities achieved maximum values as the layers accumulated to 16 and 12, respectively. As a result of certain restrictions on the angle range, there was a direct relation of the variation of the resonance angle with the sensitivity (i.e., as the variation of the resonance angle lowered, the sensitivity decreased after exceeding the optimized $\mathrm{PtSe}_{2}$ layers). In the end, we are also aware that most high-sensitivity sensors are accompanied by an increase in SPR linewidth and a reduction in figure of merit (FOM), and a higher FOM usually means a lower sensitivity [48]. Table 1 also lists the FWHM, DA, and FOM data for these $\mathrm{PtSe}_{2}$ biosensors.

Table 1. Change in thickness of $\mathrm{PtSe}_{2}$, layer numbers $(L)$, highest sensitivity $(S)$, minimum resonance angle $\left(\theta_{\min }\right)$ at $n_{s}=1.33$, and resonance angle change $(\Delta \theta)$ from $n_{s}=1.330$ to $n_{s}=1.335$. The smallest reflectivity $\left(R_{\min }\right)$, full width at half-maximum $(\mathrm{FWHM})$, and figure of merit $(\mathrm{FOM})$ is at $n_{s}=1.33$, and detection accuracy (DA) is at $n_{s}=1.33$ to $n_{s}=1.34$ for the $\mathrm{PtSe}_{2}$ SPR biochemical sensors.

\begin{tabular}{|c|c|c|c|c|c|c|c|c|c|c|}
\hline & $\mathrm{PtSe}_{2}$ & $\begin{array}{l}\text { Thickness } \\
\text { (nm) }\end{array}$ & $L$ & $\begin{array}{c}S \\
\text { (॰/RIU) }\end{array}$ & $\begin{array}{c}\theta_{\min } \\
\text { (Degree) }\end{array}$ & $\begin{array}{c}\Delta \theta \\
\text { (Degree) }\end{array}$ & $\begin{array}{l}R_{\min } \\
\text { (a. u.) }\end{array}$ & FWHM & DA & FOM \\
\hline \multirow{6}{*}{$\mathrm{Ag}$} & $\begin{array}{l}\text { Without } \\
\mathrm{PtSe}_{2}\end{array}$ & 0 & 0 & 118 & 67.64 & 0.59 & 0.0269 & 1.48 & 0.797 & 79.72 \\
\hline & \multirow{5}{*}{$\mathrm{PtSe}_{2}$} & 2.0 & 5 & 128 & 69.14 & 0.64 & 0.1096 & 3.15 & 0.4063 & 40.63 \\
\hline & & 3.3 & 9 & 139 & 70.49 & 0.69 & 0.2951 & 5.61 & 0.2477 & 24.77 \\
\hline & & 4.4 & 12 & 147 & 71.90 & 0.73 & 0.4837 & 9.17 & 0.1603 & 16.03 \\
\hline & & 5.3 & 14 & 156 & 72.84 & 0.78 & 0.5487 & 9.96 & 0.1566 & 15.66 \\
\hline & & 6.1 & 16 & 162 & 73.86 & 0.81 & 0.6043 & 10.85 & 0.1493 & 14.93 \\
\hline \multirow{4}{*}{$\mathrm{Au}$} & $\begin{array}{c}\text { Without } \\
\mathrm{PtSe}_{2}\end{array}$ & 0 & 0 & 130 & 69.57 & 0.65 & 0.0063 & 3.73 & 0.3485 & 34.85 \\
\hline & \multirow{3}{*}{$\mathrm{PtSe}_{2}$} & 2.0 & 5 & 144 & 71.40 & 0.72 & 0.0555 & 6.49 & 0.2218 & 22.18 \\
\hline & & 3.3 & 9 & 156 & 73.01 & 0.78 & 0.1808 & 9.74 & 0.1601 & 16.01 \\
\hline & & 4.4 & 12 & 165 & 74.69 & 0.82 & 0.3515 & 11.68 & 0.1412 & 14.12 \\
\hline
\end{tabular}


When the dielectric refractive index change is $\Delta n$, the reflection angle of the prism with a lower refractive index gave a larger result, which means that when the refractive index of the prism is low, a relatively higher sensitivity can be obtained [20]. Therefore, higher sensitivity can be obtained by using a prism with a low refractive index. The refractive indexes of silicon and BK7 were calculated as 3.420 and 1.515 at $\lambda=633 \mathrm{~nm}$, respectively [15,49]. Therefore, to improve the sensitivity, BK7 was chosen as the coupling prism in the proposed architecture.

Table 2 gives the information about the optimized layers of different architectures, changes in resonance angle $(\Delta \theta)$, highest sensitivity $(S)$, and FOM from another two previous papers in our laboratory. Comparing with these data of graphene, $\mathrm{MoS}_{2}$, black phosphorous heterojunction, and diversification architectures [51], $\mathrm{PtSe}_{2}$-based biosensors did not have the highest sensitivity. However, this high sensitivity and simple biosensor structure makes it easy to achieve commercial production.

Table 2. The optimized layers, change in resonance angle $(\Delta \theta)$, highest sensitivity $(S)$, and reference.

\begin{tabular}{|c|c|c|c|}
\hline Layers in the Structure & $\begin{array}{c}\Delta \theta \\
\text { (Degree) }\end{array}$ & $S(\circ /$ RIU) & Reference \\
\hline $\mathrm{Ag}, 5 \mathrm{~nm}$ black phosphorus & 0.90 & 181 & {$[50]$} \\
\hline $\mathrm{Ag}, 5 \mathrm{~nm} \mathrm{BP}, 5 \mathrm{~L}$ graphene & 1.08 & 217 & {$[50]$} \\
\hline $\mathrm{Ag}, 5 \mathrm{~nm} \mathrm{BP}, 1 \mathrm{~L} \mathrm{MoS}_{2}$ & 1.09 & 218 & [50] \\
\hline $\mathrm{Ag}, 5 \mathrm{~nm} \mathrm{BP}, 1 \mathrm{~L} \mathrm{WS}_{2}$ & 1.18 & 237 & [50] \\
\hline $\mathrm{Au}, 4 \mathrm{~L} \mathrm{MoS}_{2}, \mathrm{Au}, 1 \mathrm{~L}$ graphene & 0.91 & 182 & [20] \\
\hline $\mathrm{Ag}, 6.1 \mathrm{~nm} \mathrm{PtSe} 2$ & 0.81 & 162 & This work \\
\hline $\mathrm{Au}, 4.4 \mathrm{~nm} \mathrm{PtSe} 2$ & 0.82 & 165 & This work \\
\hline
\end{tabular}

\section{Conclusions}

On the basis of the Kretschmann configuration, we have theoretically investigated a new type of biosensor structure with the insertion of a $\mathrm{PtSe}_{2}$ layer. In this paper, SPR biochemical sensors utilizing 2D TMDC PtSe 2 have been designed and emulated to improve the sensitivities. Moreover, the various characters of $\mathrm{PtSe}_{2}$ on different metal substrates have been discussed in detail. In order to obtain a high sensitivity, we used BK7 as the coupling prism in this structure because of its low refractive index. Ag or Au films were covered with $\mathrm{PtSe}_{2}$ to enhance the sensitivities as well as the protective layers. It was found that they enhanced the sensitivities in the advised biochemical sensors, and the sensitivities of $\mathrm{PtSe}_{2}$ were $162^{\circ} / \mathrm{RIU}$ and $165^{\circ} / \mathrm{RIU}$ for $\mathrm{Ag}$ and $\mathrm{Au}$ substrates at $\mathrm{PtSe}_{2}$ layers 16 and 12 , respectively. We are convinced that these kinds of sensors can have potential applications in environmental monitoring, biological detection, medical diagnosis, chemical examination, and so on.

Author Contributions: Methodology and writing_original draft preparation, Y.J.; software, Z.L.; These two authors have equal contributions. Validation, H.W., M.S.; writing-review and editing, H.C. All authors have read and agreed to the published version of the manuscript.

Funding: This work was partially supported by the National Natural Science Foundation of China (Grant Nos. 61505111, 11604216, and 11775147) and the Science and Technology Plan Project of Shenzhen (Grant No. JCYJ20180305125443569).

Acknowledgments: This work is partially supported by the National Natural Science Foundation of China (Grant Nos. 61505111 and 11604216).

Conflicts of Interest: The authors declare no conflicts of interest.

\section{References}

1. Rothenhäusler, B.; Knoll, W. Surface-plasmon microscopy. Nature 1988, 332, 615-617.

2. Zeng, B.; Gao, Y.; Bartoli, F.J. Differentiating surface and bulk interactions in nanoplasmonic interferometric sensor arrays. Nanoscale 2015, 7, 166-170. [CrossRef] 
3. Gao, Y.; Xin, Z.; Zeng, B.; Gan, Q.; Cheng, X.; Bartoli, F.J. Plasmonic interferometric sensor arrays for high-performance label-free biomolecular detection. Lab Chip 2013, 13, 4755-4764. [CrossRef]

4. Shalabney, A.; Abdulhalim, I. Sensitivity-enhancement methods for surface plasmon sensors. Laser Photonics Rev. 2011, 5, 571-606. [CrossRef]

5. Johansen, K.; Arwin, H.; Lundström, I.; Liedberg, B. Imaging surface plasmon resonance sensor based on multiple wavelengths: Sensitivity considerations. Rev. Sci. Instrum. 2000, 71, 3530-3538. [CrossRef]

6. Ouyang, Q.; Zeng, S.; Jiang, L.; Qu, J.; Dinh, X.Q.; Qian, J.; He, S.; Coquet, P.; Yong, K.-T. Two-Dimensional Transition Metal Dichalcogenide Enhanced Phase-Sensitive Plasmonic Biosensors: Theoretical Insight. J. Phys. Chem. C 2017, 121, 6282-6289. [CrossRef]

7. Bui, V.Q.; Pham, T.T.; Le, D.A.; Thi, C.M.; Le, H.M. A firstprinciples investigation of various gas $\left(C O, \mathrm{H}_{2} \mathrm{O}\right.$, $\mathrm{NO}$, and $\mathrm{O}_{2}$ ) absorptions on a $\mathrm{WS}_{2}$ monolayer: Stability and electronic properties. J. Phys. 2015, 27, 305005.

8. Wang, H.; Zhang, H.; Dong, J.; Hu, S.; Zhu, W.; Qiu, W.; Lu, H.; Yu, J.; Guan, H.; Gao, S.; et al. Sensitivity-enhanced surface plasmon resonance sensor utilizing a tungsten disulfide $\left(\mathrm{WS}_{2}\right)$ nanosheets overlayer. Photonics Res. 2018, 6, 485-491. [CrossRef]

9. Guo, S.D. Biaxial strain tuned thermoelectric properties in monolayer PtSe 2 . J. Mater. Chem. C 2016, 4, 9366-9374. [CrossRef]

10. Wang, Z.; Li, Q.; Besenbacher, F.; Dong, M. Facile Synthesis of Single Crystal PtSe 2 Nanosheets for Nanoscale Electronics. Adv. Mater. 2016, 28, 10224-10229. [CrossRef]

11. Wang, Q.H.; Kalantar-Zadeh, K.; Kis, A.; Coleman, J.N.; Strano, M.S. Electronics and optoelectronics of two-dimensional transition metal dichalcogenides. Nat. Nanotechnol. 2012, 7, 699. [CrossRef]

12. Zhang, H.; Liu, C.-X.; Qi, X.-L.; Dai, X.; Fang, Z.; Zhang, S.-C. Topological insulators in $\mathrm{Bi}_{2} \mathrm{Se}_{3}, \mathrm{Bi}_{2} \mathrm{Te}_{3}$ and $\mathrm{Sb}_{2} \mathrm{Te}_{3}$ with a single Dirac cone on the surface. Nat. Phys. 2009, 5, 438. [CrossRef]

13. Castro Neto, A.H.; Guinea, F.; Peres, N.M.R.; Novoselov, K.S.; Geim, A.K. The electronic properties of graphene. Rev. Mod. Phys. 2009, 81, 109-162. [CrossRef]

14. Novoselov, K.S.; Geim, A.K.; Morozov, S.V.; Jiang, D.; Zhang, Y.; Dubonos, S.V.; Grigorieva, I.V.; Firsov, A.A. Electric Field Effect in Atomically Thin Carbon Films. Science 2004, 306, 666. [CrossRef]

15. Verma, R.; Gupta, B.D.; Jha, R. Sensitivity enhancement of a surface plasmon resonance based biomolecules sensor using graphene and silicon layers. Sens. Actuators B Chem. 2011, 160, 623-631. [CrossRef]

16. Saiful Islam, M.D.; Cordeiro, C.M.B.; Sultana, J.; Aoni, R.A.; Feng, S.; Ahmed, R.; Dorraki, M.; Dinovitser, A.; Ng, B.W.-H.; Abbot, D. A Hi-Bi Ultra-Sensitive Surface Plasmon Resonance Fiber Sensor. IEEE Access 2019, 7, 79085-79094. [CrossRef]

17. Saiful Islam, M.D.; Sultana, J.; Rifat, A.A.; Ahmed, R.; Dinovitser, A.; Ng, B.W.-H.; Ebendorff-Heidepriem, H.; Abbott, D. Dual-polarized highly sensitive plasmonic sensor in the visible to near-IR spectrum. Opt. Express 2018, 26, 30347-30361. [CrossRef]

18. Saiful Islam, M.D.; Sultana, J.; Aoni, R.A.; Habib, M.S.; Dinovitser, A.; Ng, B.W.-H.; Abbott, D. Localized surface plasmon resonance biosensor: An improved technique for SERS response intensification. Opt. Lett. 2019, 44, 1134-1137. [CrossRef]

19. Wu, L.; Chu, H.S.; Koh, W.S.; Li, E.P. Highly sensitive graphene biosensors based on surface plasmon resonance. Opt. Express 2010, 18, 14395-14400. [CrossRef]

20. Wu, L.; Jia, Y.; Jiang, L.; Guo, J.; Dai, X.; Xiang, Y.; Fan, D. Sensitivity Improved SPR Biosensor Based on the $\mathrm{MoS}_{2} /$ Graphene-Aluminum Hybrid Structure. J. Lightwave Technol. 2017, 35, 82-87. [CrossRef]

21. Lin, Z.; Jiang, L.; Wu, L.; Guo, J.; Dai, X.; Xiang, Y.; Fan, D. Tuning and Sensitivity Enhancement of Surface Plasmon Resonance Biosensor With Graphene Covered Au-MoS2-Au Films. IEEE Photonics J. 2016, 8, 4803308. [CrossRef]

22. Ouyang, Q.; Zeng, S.; Dinh, X.Q.; Coquet, P.; Yong, K.T. Sensitivity enhancement of $\mathrm{MoS}_{2}$ nanosheet based surface Plasmon resonance biosensor. Proc. Eng. 2016, 140, 134-139. [CrossRef]

23. Yim, C.; Lee, K.; McEvoy, N.; O’Brien, M.; Riazimehr, S.; Berner, N.C.; Cullen, C.P.; Kotakoski, J.; Meyer, J.C.; Lemme, M.C.; et al. High-Performance Hybrid Electronic Devices from Layered $\mathrm{PtSe}_{2}$ Films Grown at Low Temperature. ACS Nano 2016, 10, 9550-9558. [CrossRef]

24. Chia, X.; Adriano, A.; Lazar, P.; Sofer, Z.; Luxa, J.; Pumera, M. Layered Platinum Dichalcogenides $\left(\mathrm{PtS}_{2}\right.$, $\mathrm{PtSe}_{2}$, and $\mathrm{PtTe}_{2}$ ) Electrocatalysis: Monotonic Dependence on the Chalcogen Size. Adv. Funct. Mater. 2016, 26, 4306-4318. [CrossRef] 
25. Zhang, K.; Yan, M.; Zhang, H.; Huang, H.; Arita, M.; Sun, Z.; Duan, W.; Wu, Y.; Zhou, S. Experimental evidence for type-II Dirac semimetal in PtSe 2 . Phys. Rev. B 2017, 96, 125102. [CrossRef]

26. Wang, Y.; Li, L.; Yao, W.; Song, S.; Sun, J.T.; Pan, J.; Ren, X.; Li, C.; Okunishi, E.; Wang, Y.-Q.; et al. Monolayer $\mathrm{PtSe}_{2}$, a New Semiconducting Transition-Metal-Dichalcogenide, Epitaxially Grown by Direct Selenization of Pt. Nano Lett. 2015, 15, 4013-4018. [CrossRef]

27. Zhuang, H.L.; Hennig, R.G. Computational Search for Single-Layer Transition-Metal Dichalcogenide Photocatalysts. J. Phys. Chem. C 2013, 117, 20440-20445. [CrossRef]

28. Li, P.; Li, L.; Zeng, X.C. Tuning the electronic properties of monolayer and bilayer $\mathrm{PtSe}_{2}$ via strain engineering. J. Mater. Chem. C 2016, 4, 3106-3112. [CrossRef]

29. Lloyd, S. Graphene Flagship, AWE International. Available online: http://www.aweimagazine.com/article/ environmental-sensing-with-graphene/ (accessed on 1 October 2018).

30. Sajjad, M.; Montes, E.; Singh, N.; Schwingenschlögl, U. Superior Gas Sensing Properties of Monolayer PtSe 2 . Adv. Mater. Interfaces 2017, 4, 1600911. [CrossRef]

31. Mitsushio, M.; Miyashita, K.; Higo, M. Sensor properties and surface characterization of the metal-deposited SPR optical fiber sensors with Au, Ag, Cu, and Al. Sens. Actuators A Phys. 2006, 125, 296-303. [CrossRef]

32. Wang, L.; Sun, Y.; Wang, J.; Zhu, X.; Jia, F.; Cao, Y.; Wang, X.; Zhang, H.; Song, D. Sensitivity enhancement of SPR biosensor with silver mirror reaction on the Ag/Au film. Talanta 2009, 78, 265-269. [CrossRef]

33. Raether, H. Surface Plasmons on Smooth and Rough Surfaces and on Gratings; Springer: Berlin, Germany, 1988.

34. Cheng, Z.; Wang, Z.; Gillespie, D.E.; Lausted, C.; Zheng, Z.; Yang, M.; Zhu, J. Plain Silver Surface Plasmon Resonance for Microarray Application. Anal. Chem. 2015, 87, 1466-1469. [CrossRef]

35. Wang, Z.; Cheng, Z.; Singh, V.; Zheng, Z.; Wang, Y.; Li, S.; Song, L.; Zhu, J. Stable and Sensitive Silver Surface Plasmon Resonance Imaging Sensor Using Trilayered Metallic Structures. Anal. Chem. 2014, 86, 1430-1436. [CrossRef]

36. Yuan, C.; Lou, Z.; Wang, W.; Yang, L.; Li, Y. Synthesis of Fe3C@C from Pyrolysis of $\mathrm{Fe}_{3} \mathrm{O}_{4}$-Lignin Clusters and Its Application for Quick and Sensitive Detection of PrPSc through a Sandwich SPR Detection Assay. Int. J. Mol. Sci. 2019, 20, 741. [CrossRef]

37. Lou, Z.; Han, H.; Mao, D.; Jiang, Y.; Song, J. Qualitative and Quantitative Detection of PrPSc Based on the Controlled Release Property of Magnetic Microspheres Using Surface Plasmon Resonance (SPR). Nanomaterials 2018, 8, 107. [CrossRef]

38. Lou, Z.; Han, H.; Zhou, M.; Wan, J.; Sun, Q.; Zhou, X.; Gu, N. Fabrication of Magnetic Conjugation Clusters via Intermolecular Assembling for Ultrasensitive Surface Plasmon Resonance (SPR) Detection in a Wide Range of Concentrations. Anal. Chem. 2017, 89, 13472-13479. [CrossRef]

39. Dai, X.; Liang, Y.; Zhao, Y.; Gan, S.; Jia, Y.; Xiang, Y. Sensitivity Enhancement of a Surface Plasmon Resonance with Tin Selenide (SnSe) Allotropes. Sensors 2019, 19, 173. [CrossRef]

40. Gupta, B.D.; Sharma, A.K. Sensitivity evaluation of a multi-layered surface plasmon resonance-based fiber optic sensor: A theoretical study. Sens. Actuators B Chem. 2005, 107, 40-46. [CrossRef]

41. Xie, J.; Zhang, D.; Yan, X.Q.; Ren, M.; Zhao, X.; Liu, F.; Sun, R.; Li, X.; Li, Z.; Chen, S.; et al. Optical properties of chemical vapor deposition-grown $\mathrm{PtSe}_{2}$ characterized by spectroscopic ellipsometry. 2D Mater. 2019, 6, 035011. [CrossRef]

42. Hansen, W.N. Electric Fields Produced by the Propagation of Plane Coherent Electromagnetic Radiation in a Stratified Medium. J. Opt. Soc. Am. 1968, 58, 380-390. [CrossRef]

43. Maharana, P.K.; Jha, R. Chalcogenide prism and graphene multilayer based surface plasmon resonance affinity biosensor for high performance. Sens. Actuators B Chem. 2012, 169, 161-166. [CrossRef]

44. Homola, J. Surface Plasmon Resonance Sensors for Detection of Chemical and Biological Species. Chem. Rev. 2008, 108, 462-493. [CrossRef]

45. Zeng, S.; Baillargeat, D.; Ho, H.-P.; Yong, K.-T. Nanomaterials enhanced surface plasmon resonance for biological and chemical sensing applications. Chem. Soc. Rev. 2014, 43, 3426-3452. [CrossRef]

46. Homola, J.; YEE, S.S.; Gauglitz, G. Surface plasmon resonance sensors: Review. Sens. Actuators B Chem. 1999, 54, 3-15. [CrossRef]

47. Xiong, X. Plasmonic interface modified with graphene oxide sheets overlayer for sensitivity enhancement. ACS Appl. Mater. Interfaces 2018, 10, 34916-34923. [CrossRef] 
48. Wu, L.; Guo, J.; Wang, Q.; Lu, S.; Dai, X.; Xiang, Y.; Fan, D. Sensitivity enhancement by using few-layer blackphosphorus-graphene/TMDCs heterostructure in surface plasmonresonance biochemical sensor. Sens. Actuators B Chem. 2017, 249, 542-548. [CrossRef]

49. Maharana, P.K.; Srivastava, T.; Jha, R. On the Performance of Highly Sensitive and Accurate Graphene-on-Aluminum and Silicon-Based SPR Biosensor for Visible and Near Infrared. Plasmonics 2014, 9, 1113-1120. [CrossRef]

50. Maurya, J.B.; Prajapati, Y.K.; Singh, V.; Saini, J.P. Sensitivity enhancement of surface plasmon resonance sensor based on graphene- $\mathrm{MoS}_{2}$ hybrid structure with $\mathrm{TiO}_{2}-\mathrm{SiO}_{2}$ composite layer. Appl. Phys. A 2015, 121, 525-533. [CrossRef]

51. Lou, Z.; Wan, J.; Zhang, X.; Zhang, H.; Zhou, X.; Cheng, S.; Gu, N. Quick and sensitive SPR detection of prion disease-associated isoform (PrPSc) based on its self-assembling behavior on bare gold film andspecific interactions with aptamer-graphene oxide (AGO). Colloids Surf. B Biointerfaces 2017, 157, 31-39. [CrossRef]

C 2019 by the authors. Licensee MDPI, Basel, Switzerland. This article is an open access article distributed under the terms and conditions of the Creative Commons Attribution (CC BY) license (http://creativecommons.org/licenses/by/4.0/). 